\title{
Modelling UV irradiances on arbitrarily oriented surfaces: effects of sky obstructions
}

\author{
M. Hess and P. Koepke \\ Meteorological Institute of the L.-M. University, Munich, Germany \\ Received: 20 December 2007 - Published in Atmos. Chem. Phys. Discuss.: 18 February 2008 \\ Revised: 30 May 2008 - Accepted: 6 June 2008 - Published: 7 July 2008
}

\begin{abstract}
A method is presented to calculate UV irradiances on inclined surfaces that additionally takes into account the influence of sky obstructions caused by obstacles such as mountains, houses, trees, or umbrellas. With this method it is thus possible to calculate the impact of UV radiation on biological systems, such as, for instance, the human skin or eye, in any natural or artificial environment. The method, which consists of a combination of radiation models, is explained here and the accuracy of its results is demonstrated. The effect of a natural skyline is shown for an Alpine ski area, where the UV irradiance even on a horizontal surface may increase due to reflection from snow by more than 10 percent. In contrast, in a street canyon the irradiance on a horizontal surface is reduced to $30 \%$ in shadow and to about $75 \%$ for a position in the sun.
\end{abstract}

\section{Introduction}

UV radiation is a part of the solar spectrum with high impact on biological systems. With regard to human health, there are two conflicting effects: on the one hand, UV radiation can damage health resulting e.g. in sun burn or skin cancer, and on the other hand, the UV radiation is essential to produce vitamin D. For both effects the UV irradiance on the human skin is the basic source and thus this quantity has to be considered, namely under the conditions of the human environment. However, the UV quantity that usually is measured (Bais et al., 2007; Seckmeyer et al., 2007), derived from satellite (Arola et al., 2002; Verdebout, 2004), or forecasted (Staiger and Koepke, 2005) is the irradiance of the undisturbed upper hemisphere on a horizontal surface. Thus this quantity, in general given as UV-index (WMO, 1994), is well known in its dependence on solar elevation and atmospheric composition, and thus as function of time and position on Earth (Bais et al., 2007). In contrast, the influence of the orientation of the skin relative to the sun has hardly been considered systematically, but rather in case studies (McKenzie et al., 1997; Parisi and Kimlin, 1999; Webb, 1999; Philipona et al., 2001; Koepke and Mech, 2005). The same holds for the modification of UV radiation due to objects in the human surroundings such as walls, trees, umbrellas, or other obstructions of parts of the sky (Kimlin and Parisi, 1999; Turnbull and Parisi, 2003; Grifoni et al., 2005; Gao et al., 2002; Schween and Koepke, 2005). UV irradiances measured on different positions of the body for persons moving in their environment exist (Knuschke et al., 2007), but for a limited amount of conditions, not least due to the problems with volunteers.

Thus, for the purpose of determining systematically the influence of objects in the surroundings of humans on their UV radiation dose, we developed the method described in this paper that takes into account both the orientation of the skin and obstructions of the sky. The results presented are described as modification factors valid for erythemally weighted UV, which can be used to transform the UVI on a horizontally oriented surface into the actual UVI on a tilted receiver in an environment with obstructions of Sun or sky. This means that data of the UVI, as they are usually available, can be used to get the real UV impact. Thus all institutions or services that are interested in public health with respect to skin cancer or vitamin D production could benefit from this tool. Moreover, using other relevant weighting functions, the method also could be of value for end-users which are interested in UV irradiances in structured environments like vine yards or solar energy collector rows.

\section{Correspondence to: P. Koepke}

(peter.koepke@lmu.de)

Published by Copernicus Publications on behalf of the European Geosciences Union. 


\section{The model}

Our method is a combination of existing models, joined together by a new program. The base of the method is the radiative transfer model STAR (Ruggaber at al., 1994; Koepke et al., 2006) which yields UV radiances at each point of the sky, depending on solar elevation, atmospheric composition, and ground conditions. Next, the model Radoninc (Mech and Koepke, 2004) uses such a radiance field for given atmospheric and ground conditions to calculate UV irradiances on surfaces which may be inclined with any zenith and azimuth angle, the latter relative to the sun's position. Finally, our new program Skop deals with any objects obstructing parts of the sky. It uses the Radoninc radiances to calculate the UV-brightness of an object, includes the radiances reflected from this object into the original radiance field and then reruns Radoninc, now with the modified radiance field as input, to determine the UV irradiances on arbitrarily tilted surfaces. This whole approach allows one to introduce three dimensional effects into a one dimensional radiative transfer calculation.

The method presented here is based on solar radiation with high spectral resolution, which can be integrated to the solar radiation that is relevant for a specific UV process by using a biological action spectrum. With respect to the UV effects on humans, in the following the erythemal weighting spectrum has been used (CIE, 1987) and the results are given as UV-index (UVI), not only for the conditions of a horizontal receiver (WMO, 1994), but for all orientations as a physical quantity to describe UV irradiances (WMO, 1997).

In the current study, we model UVI on inclined surfaces with inclination zenith angles $\mathrm{ZA}$ ranging from $0^{\circ}$ to $180^{\circ}$ in steps of $10^{\circ}$, and with inclination azimuth angles AA with a $10^{\circ}$ resolution from $0^{\circ}$ to $350^{\circ}$. For zenith angles larger than $90^{\circ}$ a $30^{\circ}$ interval is applied.

\subsection{STAR}

STAR (System for Transfer of Atmospheric Radiation) is a matrix operator radiative transfer model for the UV spectral range (Ruggaber et al., 1994; Koepke et al., 2006) which took part in several model comparison activities (Koepke et al., 1998; DeBacker et al., 2001).

STAR calculates monochromatic radiances for any zenith and azimuth angle of the sky. These radiances depend on the solar elevation and azimuth angle, the state of the atmosphere, and the ground properties. The atmosphere is described by height resolved fields of the radiative properties of aerosols, clouds, and gases such as ozone and $\mathrm{SO}_{2}$. From the radiances the angularly integrated quantities irradiance and actinic flux are calculated and the values can be spectrally integrated taking into account arbitrary action spectra.

For the purposes described here, we calculate complete hemispherical radiance fields with a $1^{\circ}$ resolution in zenith and azimuth angle. The maximum spectral resolution which can be modelled is $0.2 \mathrm{~nm}$ in the UV spectral range, because it is limited by the spectral resolution of the used absorption properties of ozone and the other gases. For this study a spectral increment of $5 \mathrm{~nm}$ between $280 \mathrm{~nm}$ and $400 \mathrm{~nm}$ has been used, since the main goal is to provide modification factors which in each case are a ratio between two irradiances with the same spectral integration. For the absolute UVI the difference between these calculations and those with high, i.e. $0.5 \mathrm{~nm}$, spectral resolution is in the order of less than $1.5 \%$.

\subsection{Radoninc}

Radoninc (Radiation on Inclined Surfaces) (Mech and Koepke, 2004) uses spectral radiance fields to calculate the irradiances on arbitrarily oriented surfaces, and additionally performs the spectrally weighted integration using action spectra. To take into account the effects of the albedo of the ground around the receiver, which influences the irradiances on the tilted surface due to the photons directly reflected from the ground to this surface, a local albedo is chosen that may differ from the regional albedo which is used for the calculation of the radiation coming from the sky. Since the absorption of the UV radiation in the skin, which finally gives the relevant dose, has to be considered locally, the skin, or other biological surfaces, are divided into small areas which are assumed to be tilted, but flat. Thus in any case the radiation on the tilted surface is modelled as irradiance, taking into account the cosine weighting with respect to the local perpendicular orientation. For the erythemally weighted irradiances the results are given as UVI, also for tilted surfaces.

\subsection{Skop}

The newly developed program Skop (Sky Obstruction Program) organizes a sequence of Radoninc model runs by providing the appropriate input data. It creates input files for Radoninc, reads result files of STAR and Radoninc, creates modified radiance fields, and starts the final Radoninc runs. A detailed description of the procedure is presented here:

(a) We start with a STAR model run for the date, place, and atmospheric conditions in question. This model run yields a complete hemispheric spectrally resolved UV radiance field, using the regional albedo.

(b) Next, we select the properties of the object which is obstructing parts of the sky. Such an object may be a wall or a tree, beginning at the ground, or an umbrella or similar which obstructs only the upper parts of the sky. Receiver in the following means the human skin or the measurement device whose UV exposure will be calculated, with respect to the angles assumed as a point in the middle of the radiation field. The properties of the object are specified as: 
- Size and shape of the object in terms of its angular dimensions in zenith and azimuth, as seen from the position of the receiver (Fig. 1). The object is defined by an arbitrary number of points, given by zenith/azimuth angle combinations.

- Zenith and azimuth orientation of the object relative to the sun, under the assumption that the object is flat.

- Spectrally resolved UV-albedo, -transmission, and -emission of the object.

(c) The spectral irradiances impinging on this object are calculated by Radoninc using the radiance field from a).

(d) The radiation emerging from the object is calculated in terms of:

- The radiances reflected at the object, calculated from the irradiances on the object (cf. c)), assuming Lambertian reflectance.

- The transmitted part of the sky radiances behind the object is added if the object is transparent.

- The emitted radiances are added if the object consists of a light source.

(e) The radiation coming from the object is included into the original radiance field by replacing those sky radiances that are obstructed by the object by the radiances determined previously via d).

(f) A second Radoninc run now yields the spectral irradiances on the inclined surfaces caused by the radiance field which has been modified by the object defined in b) and the spectrally weighted irradiances like UVI.

It is possible to apply steps (b) to (e) to several objects and to include all of them in the original radiance field at the same time. In such cases, the UV brightness of each such object is determined individually. However, it has to be noted that interactions between several objects are not considered in the current version of the model. This means that there are no shadows from one object falling on the other, and that there is no reflection between such objects. We consider these effects to be of minor importance because of the usually low reflectance in the UV.

In the case of trees, partial transparency, as taken into account in the model of Schween and Koepke (2005), is a better description of the behaviour than a transmission as it is used here. However, in practice the use of a transmission is adequate due to the permanent movement of the leaves and thus a temporal variation of the spots of the transparency. If a tree has larger areas without leaves, this must of course be taken into account in the description of the obstructing object.

Clouds are not considered in the results shown below. However clouds can be easily taken into account. For overcast conditions the cloud effects can directly be considered

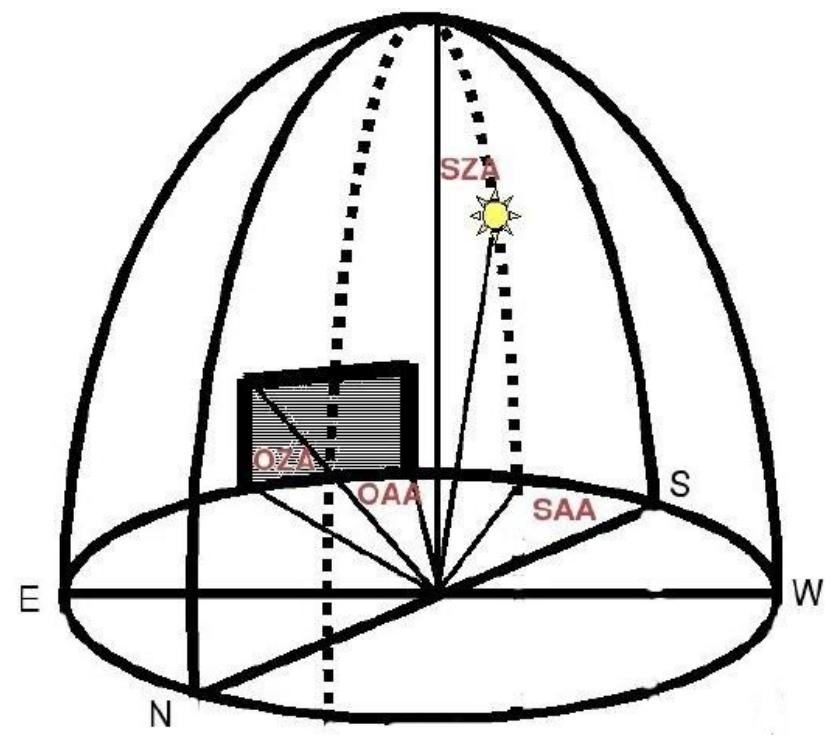

Fig. 1. Definitions of zenith and azimuth angles of sun position (SZA and SAA) and object extent (OZA and OAA). The orientation of the receiver, given with $\mathrm{ZA}$ and $\mathrm{AA}$ of its surface normal, is not shown in this figure. $\mathrm{AA}=0^{\circ}$ corresponds to South.

in STAR. Scattered clouds can be considered as other objects that obstruct parts of the sky or shade the receiver.

The model can be run on normal personal computers. The computation time is in the order of a few minutes for the final, spectrally integrated results for one scene. To compile the input properties, the geometrical properties of the objects have to be converted into angles, which is easy in most cases.

\subsection{Modification factors}

The main purpose of this paper is to describe the change of the UVI on tilted surfaces in a human environment compared to the UVI on a horizontal surface without any obstructions, as it is given in general as discussed in the introduction. Thus, we present modification factors that allow the use of such UVI on the horizontal surface without sky obstruction, for the determination of the desired UVI on a tilted surface under the specific obstruction conditions.

The change of the UVI on a tilted surface for conditions without sky obstructions has been given as the Tilt Modification Factor (Mech and Koepke, 2004), TMF, taking into account the zenith angle ZA of the tilted surface and its azimuth angle AA against sun's azimuth with $\mathrm{UVI}_{\text {hor }}$ the UVI on the horizontal surface

$\mathrm{TMF}(\mathrm{ZA}, \mathrm{AA})=\mathrm{UVI}(\mathrm{ZA}, \mathrm{AA}) / \mathrm{UVI}_{\mathrm{hor}}$

Here no sky obstructions have been taken into account, neither for the tilted nor for the horizontally oriented surface. In this paper, we introduce effects of sky obstructions 


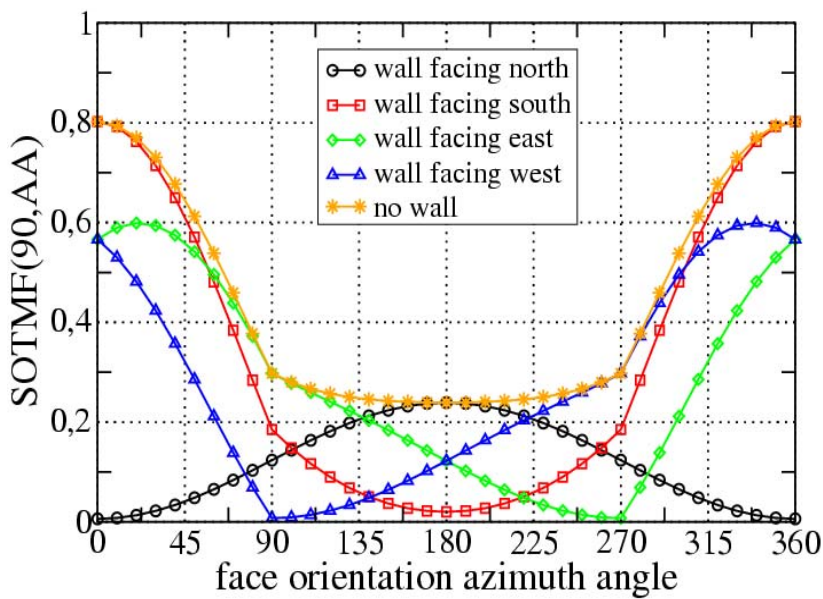

Fig. 2. Sky obstruction and tilt modification factors (SOTMF), calculated for four vertical walls facing north (black, azimuth $=180^{\circ}$ ), south (red, azimuth $=0^{\circ}$ ), east (green, azimuth $=90^{\circ}$ ), and west (blue, azimuth $=270^{\circ}$ ) respectively. The receiver surfaces are also oriented vertically $\left(\mathrm{ZA}=90^{\circ}\right)$, but rotated around the vertical axis, and thus having different face orientation azimuth angles $\mathrm{f}$. They are positioned directly in front of the walls such that the walls obstruct exactly half of the sky.

by the so called Sky Obstruction and Tilt Modification Factor, SOTMF,

$\operatorname{SOTMF}(\mathrm{ZA}, \mathrm{AA})=\mathrm{UVI}_{\mathrm{so}}(\mathrm{ZA}, \mathrm{AA}) / \mathrm{UVI}_{\text {hor }}$

Here $\mathrm{UVI}_{\mathrm{so}}(\mathrm{ZA}, \mathrm{AA})$, UVI with the subscript "so" for sky obstruction, is given for the specific conditions with sky obstruction for a receiver surface oriented towards $\mathrm{ZA}$ and $\mathrm{AA}$. UVI $\mathrm{Un}_{\text {hor }}$ here also, as always, is the UVI for conditions without sky obstruction on the horizontal surface. Thus for conditions without sky obstruction $\operatorname{SOTMF}(Z A, A A)=T M F(Z A, A A)$.

If the receiver surface is not tilted, but oriented horizontally, this is given by $\mathrm{ZA}=0$ and $\mathrm{AA}=0$ (The latter is of no relevance, but given to fulfil the nomenclature) and SOTMF

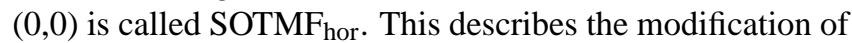
the UVI on a horizontal receiver due to sky obstruction:

$\mathrm{SOTMF}_{\text {hor }}=\mathrm{UVI}_{\mathrm{so}}(0,0) / \mathrm{UVI}_{\text {hor }}$

If a quantity is presented without azimuth angle, e.g. UVI (ZA) or SOTMF (ZA), then an average over all azimuth angles is made.

\subsection{Model verification and validation}

In order to verify the model results, and to check that the model works consistently and correctly, a number of consistency tests have been performed. One of the results is shown in Fig. 2. A vertical wall is used as an obstruction with an UV albedo of $10 \%$, in a cloud free environment with 330 DU ozone, mean aerosol turbidity, local and regional albedo

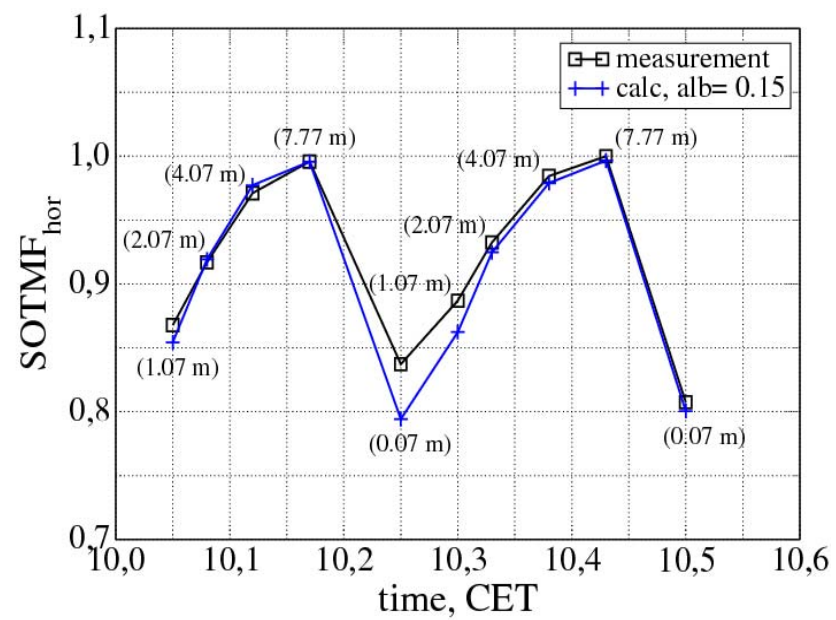

Fig. 3. Sky obstruction and tilt modification factors (SOTMF), derived from measurements in front of a wall with different distances to the wall (the distances are indicated), and for corresponding model calculations.

of $3 \%$. The receiver is a vertically oriented surface that rotates around its vertical axis, positioned directly in front of the wall. The $\mathrm{UVI}_{\text {so }}(90, \mathrm{AA})$ on this vertical receiver surface is calculated with a resolution of $10^{\circ}$ in azimuth. The position of the sun is in the south (azimuth $=0^{\circ}$ ), the solar elevation angle is $41^{\circ}$.

Figure 2 shows SOTMF (90, AA) as function of AA modelled for 4 different walls, each oriented towards one of the four cardinal directions. Each wall is assumed to obstruct half of the sky, i.e. the walls are modelled with $180^{\circ}$ width in azimuth and $90^{\circ}$ in elevation. In practice this means that the receiver is positioned directly next to a large wall. Additionally shown is SOTMF (90, AA) for conditions without a wall and thus no sky obstruction, i.e. TMF (90, AA). If the receiver surface is oriented exactly in the same direction as the wall, the radiation falling on the surface is not influenced by the wall at all, and thus SOTMF=TMF. If the receiver is looking directly towards the wall, it only receives radiation reflected from the wall, which is $10 \%$ of the radiation impinging on the wall, due to the wall's Lambertian albedo of $10 \%$. For the East and West wall cases, the highest UVI occurs at a slight angle from due south, as the receiver is turned away from the wall, which is dark because it doesn't receive radiation from the direct beam.

The validation of the model, which assures that the model is well suited to reproduce atmospheric and environmental conditions, has been achieved by comparing appropriate model calculations with UVI measurements using broadband erythemally weighting radiometers.

One example of the comparisons is presented in Fig. 3. Here we show measured and modelled SOTMF ${ }_{\text {hor }}$ for horizontally oriented receivers at different distances to a southwest oriented vertical wall on the roof of the Meteorological 
Institute in Munich. This wall extends $2.2 \mathrm{~m}$ above the instrument and has a width of $8 \mathrm{~m}$. Its UV albedo in the broad bright parts was measured to be about $15 \%$. The measurement took place on 13 April 2007 between 10:02 and 10:27 CET (Central European Time). The solar zenith angle during measurements was between $45^{\circ}$ and $48^{\circ}$. The UVI measuring device was first situated $1.07 \mathrm{~m}$ in front of the wall, and then subsequently moved to distances of $2.07 \mathrm{~m}, 4.07 \mathrm{~m}$ and $7.77 \mathrm{~m}$ away from the wall. Then, the UVI measuring device was placed directly in front of the wall at a distance of $0.07 \mathrm{~m}$, and followed by a second series of measurements with distances of 1.07, 2.07, 4.07 and $7.77 \mathrm{~m}$ from the wall. At the same time, measurements on top of this wall took place, which represent undisturbed conditions without skyline obstruction.

Figure 3 shows that modelled and measured $\mathrm{SOTMF}_{\text {hor }}$ differ only by a few percent. This is within the order of the measurement error. The measurements are sensitive to the horizontal orientation of the receiver. This holds especially true for the position close to the wall, because in this case very small tilt angles strongly affect the contribution of the wall. This effect can be seen in the data measured at 10:25 CET and 10:50 CET. In the modelled SOTMF only a small increase of the values with time can be seen, which results from the changing position of the sun, with increasing illumination of the wall and thus a reduced obscuring effect. Moreover the effective albedo of the wall, which is taken into account in the model using a fixed value, actually decreases slightly with increasing distance from the wall, since the wall has small dark brown stripes that contribute more effectively to the albedo with increasing distance.

\section{Results}

To demonstrate the model's different capabilities we show two examples. The first example describes the effect of the skyline in a natural environment, and the second example that in an urban street canyon.

\subsection{Effect of mountain skyline}

The glacier on Mount Zugspitze in Germany is situated at an altitude of about $2600 \mathrm{~m}$, which is about $300 \mathrm{~m}$ below the summit. Thus the skyline extends upwards to an elevation of more than $30^{\circ}$. The observatory Schneefernerhaus (UFS) is located in this area, and is where the Meteorological Institute performs long-term UV radiation measurements on horizontal surfaces. The measurement platform has a similar skyline as parts of the glacier, i.e. a certain part of the sky is always obstructed by the mountains. So it is of interest to determine the impact of this sky line on the UVI, especially in cases when the mountains are covered by snow. For this purpose, details of the skyline have been determined from panorama photographs. These data have been included in our model.

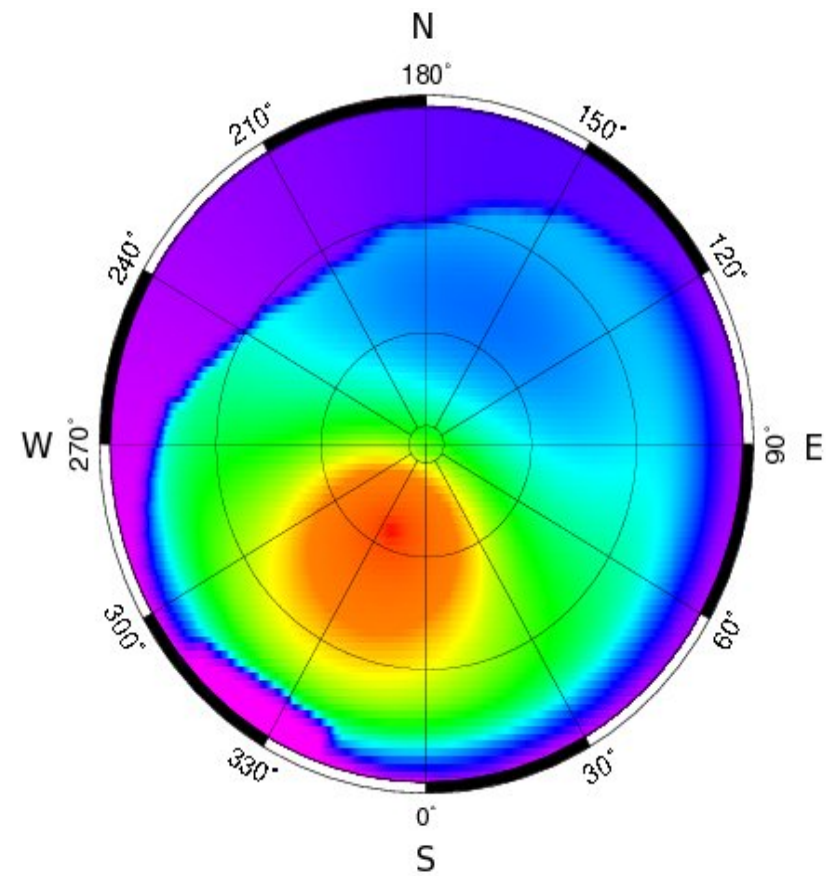

Fig. 4. Calculated UV radiances $(315 \mathrm{~nm})$ at Schneefernerhaus at 21 June, 13:00 CET with skyline as seen from the measurement platform. The figure shows an intermediate result of our model, representing step e) from Sect. 2.3. The mountains have been included in the original STAR-radiance field, accounting as well for their angular extent as for their UV brightness determined by Radoninc, steps b)-d). The rainbow colour scale represents radiances, non-linearly distributed between (violet) 0.024 and (red) $0.412 \mathrm{~W} \mathrm{~m}^{-2} \mathrm{sr}^{-1} \mathrm{~nm}^{-1}$.

The skyline is composed by 72 rectangles, each with a width of $5^{\circ}$ in azimuth AA, with different height (ZA), and a slope of $45^{\circ}$ in zenith in each case. The skyline is shown in Fig. 4 in violet. In this figure the outer angle describes the azimuth $\mathrm{AA}$, where $0^{\circ}$ is south. The radial distance represents the zenith angle $\mathrm{ZA}$, with $0^{\circ}$ in the centre and $90^{\circ}$ at the border. The two circles show $\mathrm{ZA}=30^{\circ}$ and $60^{\circ}$.

The colours in Fig. 4 represent the radiances modelled for a solar zenith angle of $25.4^{\circ}$ and a solar azimuth of $336^{\circ}$ (south at $0^{\circ} / 360^{\circ}$ ), as valid for 21 June at 13:00 CET. The atmosphere is modelled with $330 \mathrm{DU}$ ozone, 0.1 aerosol optical depth at $500 \mathrm{~nm}$ and continental average as aerosol type (Hess et al., 1998). A regional albedo of $3 \%$ is specified. A rainbow colour scale, reaching from violet (low values) to red (high values) is used to show UV radiances at $315 \mathrm{~nm}$, non-linearly distributed between 0.024 and 0.412 $\left(\mathrm{W} \mathrm{m}^{-2} \mathrm{sr}^{-1} \mathrm{~nm}^{-1}\right)$. The direct Sun is not included in the data, but the maximum of the scattered radiances is at the position of the sun, i.e. the red area shifted towards the zenith within the orange area, which shows the bright part of the sky in the UV. It is clear to see that the mountains are dark (violet to blue) in comparison to the sky, but are brightest 


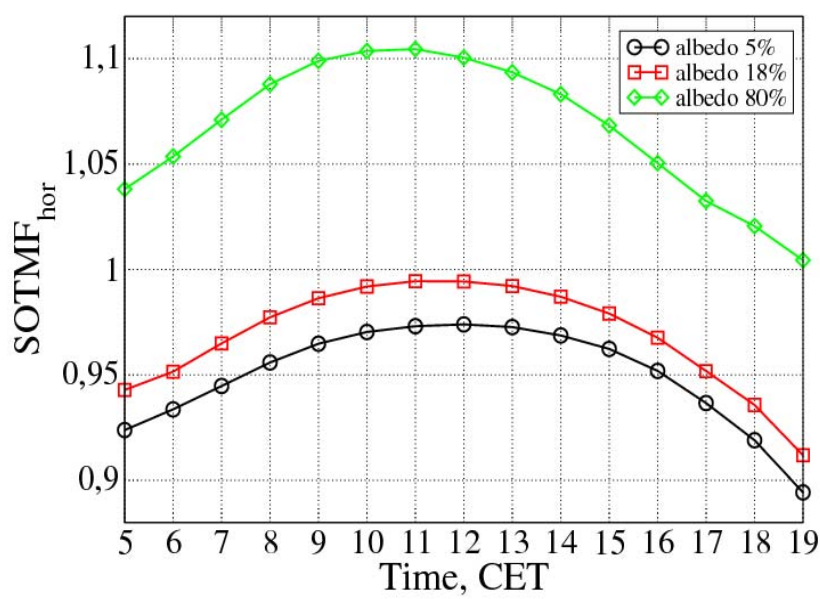

Fig. 5. Sky obstruction and tilt modification factors (SOTMF) on horizontal surfaces for the diurnal variation at Schneefernerhaus on 21 June. Three values of local surface albedo are considered for the region around Schneefernerhaus.

(blue) in the region opposite the sun's position, since here the radiation impinges nearly perpendicularly on the surface. In other azimuth directions the angle of incidence relative to the mountain surface is larger, which results in lower reflected UV radiation.

In Fig. 5, the calculated diurnal variation of the SOTMF at Schneefernerhaus is shown for June 21 for the atmospheric conditions mentioned above and three assumptions of the mountain albedo. Albedos of $5 \%$ and $18 \%$ are assumed for summer conditions. The $18 \%$ value has been measured to be the albedo of rocks around Schneefernerhaus (Bucars, 2006), and $5 \%$ is a value taken from literature (Blumthaler and Ambach, 1988). Secondly, an albedo of $80 \%$ has been chosen to model snow conditions (Grenfell and Warren, 1994). Although Mount Zugspitze generally is not covered with snow on 21 June, this calculation is intended to show the sensitivity of the UVI on the albedo of the obstructing parts of the horizon for a wide range of solar zenith angles. The regional albedo in all cases is assumed to be 3\%, indicating no snow in the surrounding wide area below the mountain. In case of the two lower albedo values, the SOTMF than 1, i.e. the skyline reduces the UVI. This reduction is less than $5 \%$ between 8 a.m. and 4 p.m., when the absolute UVI is larger than 2, and thus within the range of the measurement uncertainties. But it is of course a systematic deviation. In case of snow cover, the mountains are brighter than the parts of the sky that they obstruct, and thus the UVI is up to $10 \%$ larger than without the sky obstruction. The asymmetry of SOTMF $_{\text {hor }}$ in its daily course results from the mountain influence which varies with azimuth. For the assumption of snow this effect shifts the maximum value towards 11:00 CET, as a consequence of the maximum mountain influence at about $210^{\circ}$. Therefore, the UVI measurements at Schneeferner-

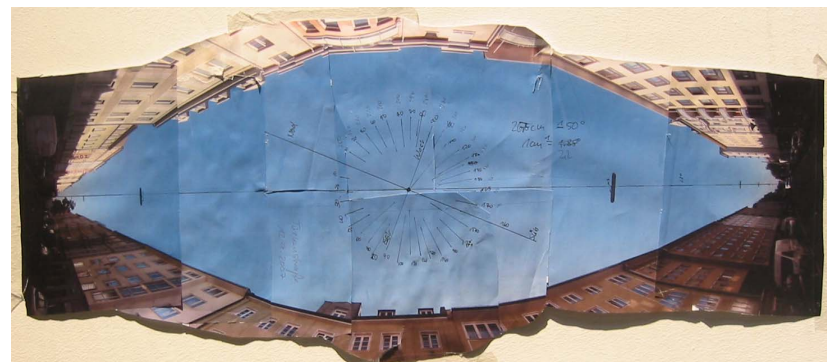

Fig. 6. Composition of photographs for representation of the street canyon Türkenstraße in Munich. The street is oriented from SE to $\mathrm{NW}$ and has a width of $18 \mathrm{~m}$. The typical height of the houses is also about $18 \mathrm{~m}$.

haus are not significantly affected by the skyline in summer, but may be up to $10 \%$ too high during winter. This is valid for a horizontally oriented receiver. For the skin of people on the glacier (tilted surfaces) the effect of snow can be much larger, up to 70\% (Philipona et al., 2001; Koepke and Mech, 2005).

\subsection{Street canyon}

A majority of people spend most of their time in cities. Even if they leave the house, their gain of UV radiation is always reduced compared to an open environment, because the buildings obstruct parts of the sky.

As an example of a typical urban street canyon, Türkenstraße in Munich is used. The angular extent of the houses has been estimated from a series of photographs (Fig. 6). The width of the street is $18 \mathrm{~m}$ and the typical height of the five-storey houses is also about $18 \mathrm{~m}$. The resulting skyline, as used in the subsequent model calculations, varies with $\mathrm{ZA}=90^{\circ}$ as a maximum for the azimuths where the street meets the horizon and minimum of $\mathrm{ZA}$ of $32^{\circ}$ for the position perpendicular to the observer. The street is oriented by about $15^{\circ}$ in SE-NW direction, as indicated in Fig. 6.

The modelled variations of UVI (ZA), $\mathrm{UVI}_{\mathrm{so}}(\mathrm{ZA})$ and SOTMF (ZA) are shown in Fig. 7a, b, c as function of time for a cloud free summer day (21 June), with 330 DU ozone, 0.1 aerosol optical depth at $500 \mathrm{~nm}$ and continental average as aerosol type (Hess et al., 1998), and a regional albedo $3 \%$. Modelling is made under the assumption that people are moving, i.e. the values are averaged over the orientation azimuth angles of the receiver surfaces. The values are given for fixed hours only, linearly connected to guide the eye.

Figure 7a shows UVI(ZA), i.e. under the assumption of no obstructions. The typical daily course can be seen where irradiance increases with decreasing solar zenith angle, and a maximum at local noon at about 12:15 CET. The UVI(ZA) decreases with increasing slope of the receiver, ZA, due to the contribution of sun and sky being reduced and replaced by lower radiances reflected at the ground. 


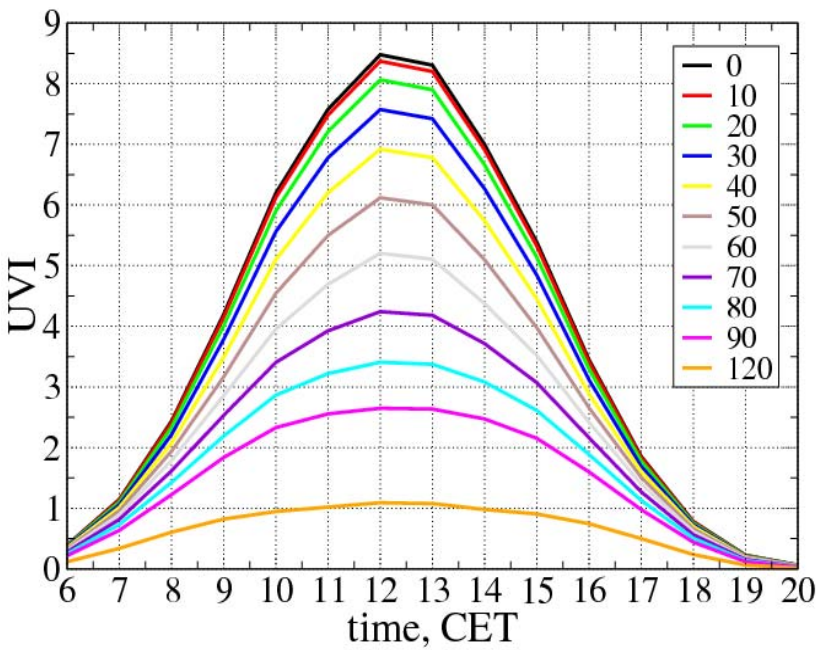

Fig. 7a. UVI(ZA) on inclined surfaces, averaged over all orientation azimuth angles. Zenith angles are given as lines with different colours. These data are modelled for Munich under the assumption of climatological mean values of the atmospheric conditions in June. No skyline effects are considered.

Figure $7 \mathrm{~b}$ shows the $\mathrm{UVI}_{\mathrm{so}}(\mathrm{ZA})$ for a receiver in the center of the street canyon, i.e. taking into account the obstruction of sky and sun due to the buildings. All values are reduced considerably against the conditions with free horizon, with a big step between 10:00 and 11:00 CET, when the sun begins to shine into the street and the receiver comes out of shadow. The asymmetry against local noon results from the SE-NW direction of the street, meaning that the sun shines parallel to the street at about 13:00 CET.

The effect of the obstructions is presented in Fig. 7c as $\operatorname{SOTMF(ZA).~During~morning~and~afternoon,~when~the~}$ street is in shadow, SOTMF is less than $30 \%$. But even without shadow, during the time when the sun shines into the street, the $\mathrm{UVI}_{\text {so }}(\mathrm{ZA})$ is reduced to $75 \%$ or less compared to the undisturbed conditions. The effect is lowest for the surfaces looking towards the sky, i.e. for the small ZA. For ZA larger than $50^{\circ}$ the SOTMF(ZA) decreases strongly with increasing $\mathrm{ZA}$, and is reduced to values of $50 \%$ for sunny and $20 \%$ for shadow conditions for vertically oriented surfaces $\left(\mathrm{ZA}=90^{\circ}\right)$ like parts of the skin. For the conditions with shadow at the receiver position, the obstruction effect decreases with increasing solar zenith angle, because the relative contribution of the unobstructed sky near the zenith to the global irradiance is increased. The $\operatorname{SOTMF}(\mathrm{ZA})$ are nearly constant as long as the sun is shining into the street canyon. This effect results from the relative contribution of sun and sky that does nearly not change with time compared to the undisturbed conditions and due to the relatively low contribution of the buildings due to their low albedo.

The SOTMF(ZA) are only very slightly influenced by aerosol optical depth and ozone amount (not shown) since these quantities affect the absolute UVI, independent of whether obstructions are present or not.

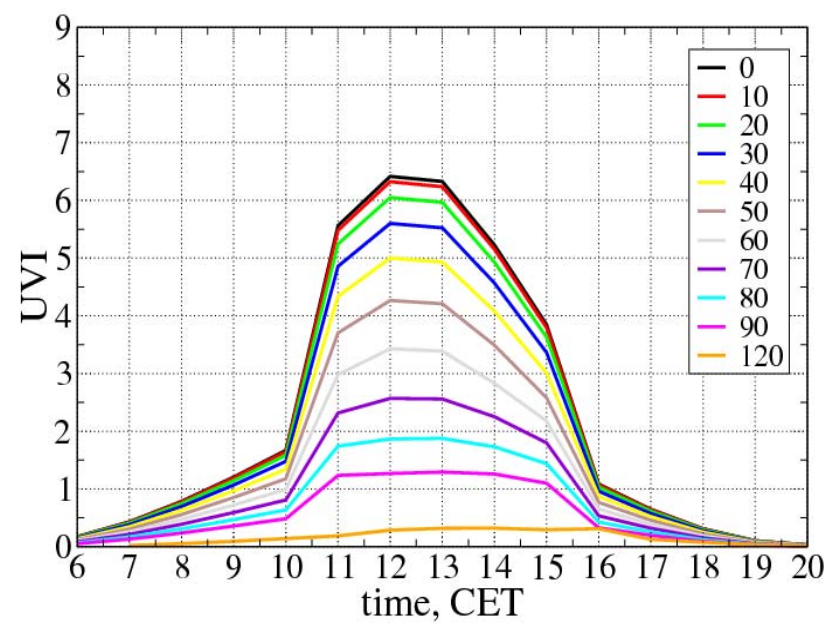

Fig. 7b. As Fig. 7a, but in the middle of the street canyon from Fig. 6.

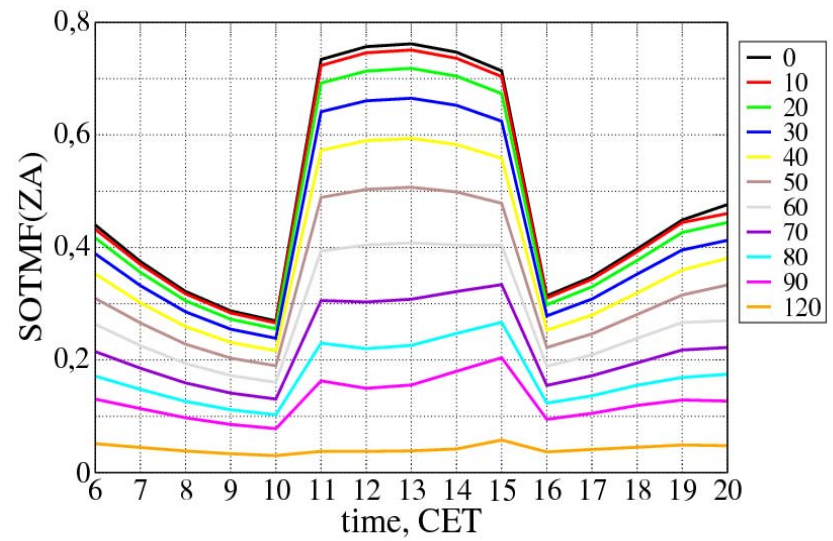

Fig. 7c. SOTMF calculated from Fig. 7a and b. These modification factors show the effect of the Munich street canyon on UVI on inclined surfaces, averaged over all orientation azimuth angles. Zenith angles are given as lines with different colours. These data are modelled for Munich under the assumption of climatological mean values of the atmospheric conditions in June.

\section{Conclusions}

A method has been presented which allows one to model spectrally weighted UV irradiances for a wide range of applications relevant to biological systems.

We are able to perform calculations for all atmospheric conditions given by solar zenith and azimuth angle, surface albedo, surface topography, and atmospheric constituents such as ozone concentration, aerosol amount and composition, and clouds. The resulting UV irradiances may be weighted by any spectral response function. Furthermore, the surfaces where the radiation is impinging on may be tilted by any angle in zenith and azimuth directions. This feature allows modelling the UV irradiance for the human 
skin or eye. The new feature of the method is the consideration of objects obstructing parts of the sky and thus reducing the diffuse UV radiation, and also the direct solar beam, i.e. shadow. These obstructions may be of any size and shape, and they may have individual reflection, transmission and emission properties. So it is possible to model the diverse human environment, as well the effects of objects forming a skyline such as walls, trees, street canyons or mountains, as the effects of shadows caused by umbrellas, awnings or trees.

Since different biological weighting functions can be used, both positive (vitamin D) and negative (sun burn, skin cancer) aspects of UV irradiance can be analysed with respect to public health. The model can be used also to analyse UV effects for plants (e.g. wine yards) or artificial surfaces (e.g. weathering of solar panels). The determination of modification factors gives the possibility to use the results in combination with currently measured UVI on a horizontal surface.

The model will be compared with measurements made for a wide range of radiation environments and be used for sensitivity studies, to give modification factors valid for average conditions.

Acknowledgements. This study has been funded by the Bavarian State Ministry of the Environment, Public Health and Consumer Protection.

Edited by: M. Blumthaler

\section{References}

Arola, A., Kalliskota, S., den Outer, P.N., Edvardsen, K., Hansen, G., Koskela, T., martin, T.J., Matthijsen, J., Meerkötter, R., Peeters, P., Seckmeyer, G., Simon, P., Slaper, H., Taalas, P., and Verdebout, J.: Four UV mapping procedures using satellite data and their validation against ground-based UV measurements, K. Geophys. Res., 107, ACL11, 1-11, 2002.

Bais, A., Lubin, D., Arola, A., Bernhard, G., Blumthaler, M., Chubarova, N., Erlick, C., Gies, H. P., Krotkov, N., Lantz, K., Mayer, B., McKenzie, R.L., Piacentini, R. D., Seckmeyer, G., Slusser, J. R., and Zerefos, C. S.: Surface ultraviolet radiation: Past, present, and future, Chapter 7 in: Scientific Assesment of Ozone Deplition: 2006. Global ozone and Monotoring Project. Report No. 50, World Meteorological organization, Geneva, 7.17.53, 2007.

Blumthaler, M. and Ambach, W.: Solar UVB albedo of various surfaces, Photochem. Photobiol, 48(1), 85-88, 1988

Bucars, N.: Regionale und lokale Albedo im UV-Spektralbereich, Diploma-thesis, Meteorolog. Inst. Univ. Munich, 46 p., 2006.

CIE: A reference action spectrum for ultraviolet induced erythemal in human skin, CIE research note, CIE Journal 6, 17-22, 1987.

DeBacker, H., Koepke, P., Bais, A., de Cabo, X., Frei, T., Gillotay, D., haite, C., Heikkilä, A., Kaqzantzidis, A., Koskela, T., Kyrö, E., Lapeta, b., Lorente, J., Masson, K., Mayer, B., Plets, H., Redondas, A., Renaud, A., Schauberger, G., Schmalwieser, G., Schwander, H., Vanicek, K.: Comparison of measured and mod- eled UV indices for the assessment of health risks, Meteorol. Appl., 8, 267-277, 2001.

Gao, W., Grant, R. H., Heisler, G. M., Slusser, J. R.: A Geometric Ultraviolet-B Radiation Transfer Model Applied to Vegetation Canopies, Agron. J., 94, 475-482, 2002.

Grenfell, T. C. and Warren, S. G.: Reflection of solar radiation by the Antarctic snow surface at ultraviolet, visible, and nearinfrared wavelengths, J. Geophys. Res., 99(D6), 18 669-18 684, 1994.

Grifoni, D., Carreras, G., Sabatini, F., and Zipoli, G.: UV hazard on a summertime day under Mediterranean conditions and protective role of a beach umbrella, Int. J. Biometeorolog., 50 (2), 75-82, 2005.

Hess, M., Koepke, P., and Schult, I.: Optical properties of aerosols and clouds: the software package OPAC, Bull. Am. Met. Soc., 79, 831-844, 1998.

Kimlin, M. G. and Parisi, A. V.: Comparison of the spectral biologically effective solar ultraviolet in adjacent tree shade and sun, Phys. Med. Biol., 44, 2071-2080, 1999.

Knuschke, P., Unverricht, I., Ott, G., and Janssen, M.: Personenbezogene Messung der UV-Exposition von Arbeitnehmern im Freien, Abschlussbericht zum Projekt F1777 im Auftrag der Bundesanstalt für Arbeitsschutz und Arbeitsmedizin, 2007.

Koepke, P., Bais, A., Balis, D., Buchwitz, M., Backer, H.D., Cabo, X.D., Eckert, P., Erikson, P., Gillotay, D., Heikkilä, A., Koskela, T., Lapeta, B., Litynska, Z., Lorente, J., Mayer, B., Renaud, A., Ruggaber, A., Schauberger, G., Seckmeyer, G., Seifert, P., Schmalwieser, A., Schwander, H., Vanicek, K., and Weber, M.: Comparison of models used for UV Index calculations, Photochem. Photobiol., 67(6), 657-662, 1998.

Koepke, P. and Mech, M.: UV irradiance on arbitrarily oriented surfaces: variation with atmospheric and ground properties, Theor. Appl. Climatol., 81, 25-32, 2005.

Koepke, P., Anwender, D., Mech, M., Oppenrieder, A., Reuder, J., Ruggaber, A., Schreier, M., Schwander, H., and Schween, J.: Actual state of the UV radiation transfer model package STAR, in Fischer and Sohn (Eds.) IRS2004: Current Problems in Atmospheric Radiation, A. Deepak Publ., Hampton, USA, 71-74, 2006.

Mc Kenzie, R. L., Paulin, K. J., and Kotkamp, M.: Erythemal UV Irradiances at Lauder, New Zealand: Relationship between horizontal and normal incidence, Photochem. Photobiol., 66(5) 683689, 1997.

Mech, M. and Koepke, P.: Model for UV irradiance on arbitrarily oriented surfaces, Theor. Appl. Climatol., 77, 151-158, 2004.

Parisi, A. V. and Kimlin, M. G., Horizontal and sun-normal spectral biologically effective ultraviolet irradiances. J Photochem. Photobiol. B: Biol, 53, 70-74, 1999.

Philipona R., Schilling A., and Schmucki D.: Albedo enhanced maximum UV irradiance measured on surfaces orientated normal to the Sun. Photochem. Photobiol. 73(4), 366-375, 2001

Ruggaber, A., Dlugi, R., and Nakajima, T.: Modelling radiation quantities and photolysis frequencies in the troposphere, J. Atmos. Chem., 18, 171-210, 1994.

Schween, J. and Koepke, P.: Modelling the UV-exposure within a plant stand during a vegetation period, Meteor. Z., 14(2), 129135, 2005.

Seckmeyer, G., Pisulla, D., Glandorf, M., HENRIQUES; D., Johnsen, B., Webb, A., Siani, A:M. Bais, A., Kjeldstad, B., Brog- 
niez, C., Lenoble, J., Gardiner, B., Kirsch, P., Koskela, T., Kaurola, J., Uhlmann, B., Slaper, H., den Outer, P., Janouch, M., Werle, P., Gröbner, J., Mayer, B., de la Casiniere, A., Simic, S., and Carvalho, F.: Variability of UV irradiance in Europe, Photochem. Photobiol., 83, 1-8, 2007.

Staiger, H. and Koepke, P.: UV index forecasting on a global scale, Meteorolog. Zeitschrift, 14(2) 259-270, 2005.

Turnbull, D. and Parisi, A. V.: Spectral UV in public shade settings, J. Photochem. Photbiol.: B Biol., 69, 13-19, 2003.

Verdebout, J.: A European satellite-derived UV climatology available for impact studies, Radiat. Prot. Dosim, 111(4), 407-411, 2004.
Webb, A. R, Weihs, P., and Blumthaler, M.: Spectral UV irradiance on vertical surfaces: a case study. Photochem Photobiol, 69(4), 464-470, 1999.

WMO: Report of the WMO meeting of experts on UVB measurements, data quality and standardization of UV indices. Report 95, World Meteorological Organization, Geneva, Switzerland. Les Diablerets, 1994.

WMO: Report of the WMO-WHO meeting of experts on standardization of UV indices and their dissemination to the public. Report 127, World Meteorological Organization, Geneva, Switzerland. Les Diablerets, 1997. 